\title{
Impact of COVID-19 on Patient Trends and Outcome: Results from a Tertiary Care Burn and Plastic Unit in Northern India
}

\author{
Nupur Aggarwal ${ }^{1, \odot ~ R a k e s h ~ K u m a r ~ S r i v a s t a v a ~}{ }^{1}$ \\ ${ }^{1}$ Department of Burns, Plastic and Maxillofacial Surgery, Vardhman \\ Mahavir Medical College and Safdarjung Hospital, New Delhi, India \\ Address for correspondence Nupur Aggarwal, MBBS, MS, \\ Department of Burns, Plastic and Maxillofacial Surgery, Vardhman \\ Mahavir Medical College and Safdarjung Hospital, Ansari Nagar, \\ New Delhi 110029, India (e-mail: nupur_gzb@yahoo.co.in).
}

\begin{abstract}
Keywords

- COVID-19

- burn injuries

- trends

- impact

- outcome

- effect

- risk factors

- mortality

Background Burn and trauma injuries need emergency care and resuscitation, which required uninterrupted delivery of inpatient care services during the coronavirus disease 2019 (COVID-19) pandemic. Burn patients are physiologically immunocompromised, increasing the risk of COVID-19 infection in them. This study analyzes the impact of COVID-19 pandemic on patient trends in a burn and plastic unit and assesses the effect of COVID-19 infection in burns.

Methods This single-center, retrospective observational case-control study was conducted in the Department of Burns, Plastic and Maxillofacial Surgery of a tertiary care hospital in New Delhi, India. Patient data was collected from April 1, 2019 to August 10, 2019 and from April 1, 2020 to August 10, 2020. All data of burns and trauma patients collected was analyzed and compared.

Results There were total 350 admissions during COVID time period and 562 admissions during non-COVID time period. The admission rate, type of burn injury, and death rate did not vary significantly during the two time periods. Thermal burn was the most common type of burn injury. There were total 18 cases diagnosed to be COVID-19 positive during the pandemic. There were two deaths among COVID-19 positive burn cases. Conclusion This study finds no difference in patient patterns during COVID and non-COVID time period. Amongst burn patients, no increased risk of COVID-19 infection is seen with larger body surface area of burns. No increase in mortality is seen in burn patients infected with COVID-19.
\end{abstract}

\section{Introduction}

In 2020, the worldwide surge of coronavirus disease of 2019 (COVID-19) infection has set social distancing, restricted social activity, work from home and use of face-masks as the new normal for safe and healthy living. Hospital footfall of patients for non-COVID complaints saw a major decline due to mobility restrictions as well as fear of contracting infection from hospitals. However, burn and trauma injuries are difficult to be managed at home and mandated continuation of inpatient hospital care services with necessary alterations against COVID-19 infection, during the pandemic. ${ }^{1}$

(C) 2021. Association of Plastic Surgeons of India.

This is an open access article published by Thieme under the terms of the Creative Commons Attribution-NonDerivative-NonCommercial-License, permitting copying and reproduction so long as the original work is given appropriate credit. Contents may not be used for commercial purposes, or adapted, remixed, transformed or built upon. (https://creativecommons.org/licenses/by-nc-nd/4.0/).

Thieme Medical and Scientific Publishers Pvt. Ltd. A-12, 2nd Floor, Sector 2, Noida-201301 UP, India 
Burn injury is an acute emergency that demands an early initiation of treatment without any delay. Moreover, burn patients are in a state of hypermetabolism, hyperdynamic circulation, and are at a high risk of developing systemic inflammatory response syndrome (SIRS), i.e multi-organ dysfunction, respiratory failure, hypotension, and thrombocytopenia followed by mortality. ${ }^{2}$ Burn patients are also physiologically immunocompromised, which adds to their vulnerability if they contract COVID-19 infection.,4

Alteration in the epidemiology of burns is expected during the COVID pandemic due to change in the pattern of daily activities. To improve the outcome of burns during this pandemic, we needed to evaluate the incidence, risk factors, and outcome of COVID infection in burn patients. This will help us in future formulation of prevention and treatment guidelines for COVID-19 infection in burn patients.

In this study, we compare the trends and outcome of burn and trauma patients between COVID and non-COVID study time period. We evaluate percentage of total body surface area (TBSA) of burns as a risk factor for increased chances of COVID-19 infection in burns. The outcome of COVID-19 infection in burns in terms of mortality has also been assessed.

\section{Materials and Methods}

\section{Study Design}

This single-center, retrospective observational case-control study was conducted in the Department of Burns, Plastic and Maxillofacial Surgery of a tertiary care hospital in New Delhi, India. All data of burn and trauma patients admitted from April 1, 2020 to August 10, 2020 was collected. Similarly, patient data from April 1, 2019 to August 10, 2019 was collected for comparison with patient characteristics during normal times. The clinical course of COVID-19-positive patients was also observed.

\section{Statistical Analysis}

Descriptive statistics of data was presented in terms of frequency/percentage for qualitative variables. Data was tested for normality and accordingly, continuous variables were expressed as range (min, max)/ mean \pm standard deviation/median \pm interquartile range. The statistical significance of quantitative variables between COVID and
non-COVID time period was done using nonparametric Mann-Whitney U test.

The case-control analysis for TBSA of burns as a risk factor for COVID-19 infection in burns included 16 cases of burns with COVID-19 infection. Case was defined as a burn patient with COVID-19 infection confirmed by reverse transcription-polymerase chain reaction (RT-PCR) testing. Sixteen controls were selected from burn patients without COVID-19 infection during 2020 study time period. Cases and controls were matched for age and gender. Independent sample $t$-test was used for testing statistical significance.

The case-control analysis for outcome of COVID-19 infection in burns as mortality included 14 cases of burns with COVID-19 infection (two positive cases refused treatment and could not be followed up). The definition of cases and controls remained same as previously described. Fourteen controls were selected from burn patient data during 2019 study time period. Cases and controls were matched for age ( \pm 4years), percentage TBSA of burns ( $\pm 5 \%$ ), and gender. Fisher's exact test was used for testing statistical significance.

SPSS version 26.0, IBP Corp., Armonk, New York, United States was used. Significance was set as $p<0.05$ with $95 \%$ confidence intervals.

\section{Results}

There were total 350 admissions from period of April 1, 2020 to August 10, 2020 and 562 admissions from period of April 1, 2019 to August 10, 2019. The comparison of demographic data of patients admitted during COVID and non-COVID time period is shown in - Table 1 .

There were 318 burn cases (90.9\%) and 32 trauma cases (9.1\%) during the COVID time period with 557 burn cases (98.9\%) and only 5 trauma cases $(0.1 \%)$ during non-COVID time period. There were $51.7 \%$ thermal burns, $18.9 \%$ electric contact burns, $5.4 \%$ electric flash burns, $13.7 \%$ scalds, and $1.1 \%$ chemical burns during COVID time period. During non-COVID time period, $56.84 \%$ thermal burns, $15.28 \%$ electric contact burns, $7.1 \%$ electric flash burns, $17.76 \%$ scalds, and $1.95 \%$ chemical burns were recorded. Comparison of trends of admissions during the COVID and non-COVID time period is shown in - Fig. 1. No significant difference was found in admission trends between the two study periods $(p=0.317)$.

Table 1 Comparison of demographic data between COVID and non-COVID time period

\begin{tabular}{|l|l|l|l|}
\hline & COVID time period & Non-COVID time period & $p$-Value \\
\hline No. of admissions & 350 & 562 & 0.317 \\
\hline No. of pediatric (<16 y) cases (\%) & $114(32.57 \%)$ & $161(28.65 \%)$ & 0.317 \\
\hline Mean age & $25 \pm 0.92$ y (range: $1-90$ y) & $25.5 \pm 17.7$ y (range: $1-88$ y) & 0.317 \\
\hline Mean TBSA of burns & $\begin{array}{l}41.7 \pm 1.4 \% \\
(\text { range: } 2-98 \%, \text { median: } 35 \%)\end{array}$ & $\begin{array}{l}43.7 \pm 27.4 \%(\text { range: } 1-99 \%, \\
\text { median: } 40 \%)\end{array}$ & 0.317 \\
\hline Male:female ratio & $230: 120$ & $306: 256$ & 0.317 \\
\hline No. of deaths $(\%)$ & $55(15.71 \%)$ & $196(34.88 \%)$ & 0.317 \\
\hline
\end{tabular}

Abbreviations: COVID-19, coronavirus disease 2019; TBSA, total body surface area. 


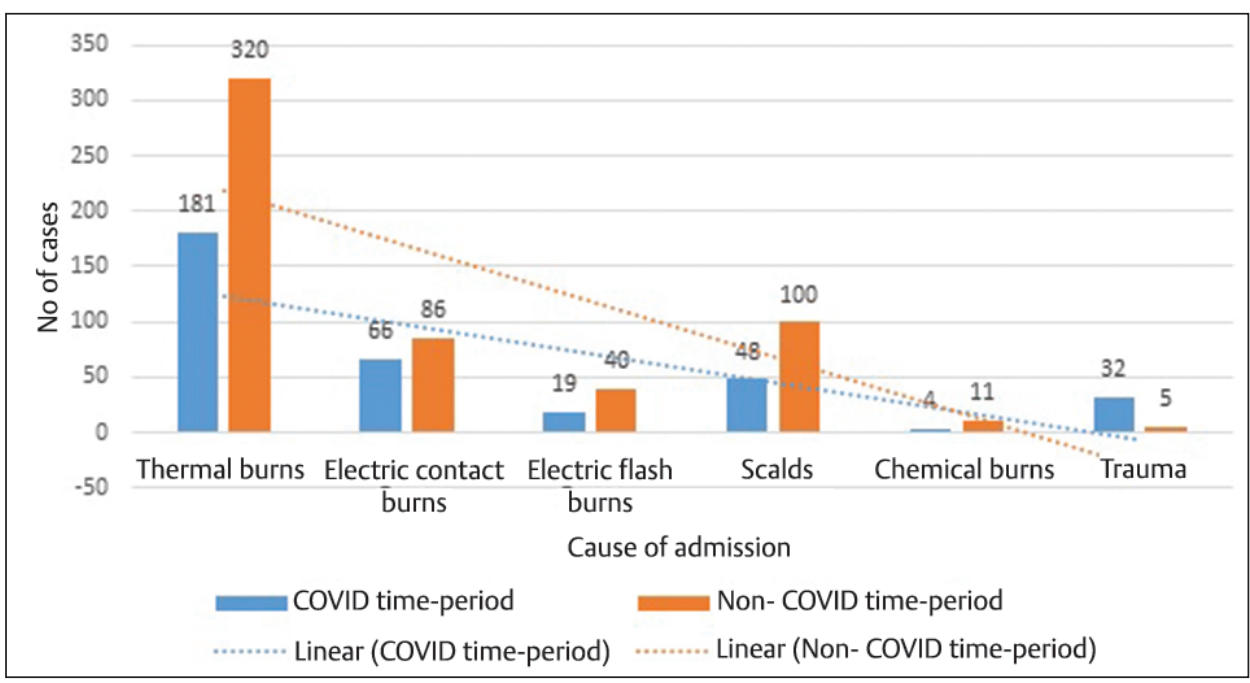

Fig. 1 Trend of admissions in burn and plastic unit during coronavirus disease 2019 (COVID) and non-COVID time period.

Around $62.6 \%$ cases (199 out of 318 burn cases) had TBSA of burns greater than 25\% during COVID time period and 63.9\% cases (356 out of 557 burn cases) during non-COVID time period. Distribution of TBSA of burns between two groups is shown in - Table 2.

There were total 18 patients who were diagnosed to be COVID-19 positive using RT-PCR testing. Of these, 15 patients were new admissions and 3 were already inpatient, who were diagnosed to be COVID-19 positive on screening. Out of 18 COVID-19 infected patients, there were 16 burn cases and 2 trauma cases. These 16 COVID-19 infected burn cases were included in the study for further analysis. The mean age of cases was $(20.63 \pm 13.15)$ years. There were 10 males and 6 females among the cases. Mean TBSA of burns in the case group was $(30.94 \pm 13.44) \%$. Thermal burn was present in $52.9 \%$ cases (9 out of 16 ). Rest there were two cases of electric contact burn, two scalds, and one case of electric flash burn.

On analysis of 16 COVID-19-positive cases against 16 matched controls identified from COVID time period, no increased risk of COVID-19 infection in burn patients with

Table 2 Distribution of TBSA of burns between COVID and non-COVID time period

\begin{tabular}{|l|l|l|}
\hline & $\begin{array}{l}\text { COVID time } \\
\text { period }\end{array}$ & $\begin{array}{l}\text { Non-COVID time } \\
\text { period }\end{array}$ \\
\hline TBSA of burns & No. of cases (\%) & No. of cases (\%) \\
\hline $0-25 \%$ & $119(37.4 \%)$ & $201(36.1 \%)$ \\
\hline $25-50 \%$ & $98(30.8 \%)$ & $171(30.7 \%)$ \\
\hline $50-75 \%$ & $60(18.9 \%)$ & $91(16.3 \%)$ \\
\hline $75-100 \%$ & $41(12.9 \%)$ & $94(16.9 \%)$ \\
\hline Total & 318 & 557 \\
\hline
\end{tabular}

Abbreviations: COVID-19, coronavirus disease 2019; TBSA, total body surface area.

Notes: Total number of cases with burns in COVID time period (thermal + scald + electric contact + electric flash + chemical) $=318$; total number of cases with burns in non-COVID time period (thermal + scald + electric contact + electric flash + chemical) $=557$. larger surface area of burns was seen $(p=0.126)$. Around $56.4 \%$ COVID-19 infected cases had TBSA of burns $>25 \%$ which is not much different from non-COVID-19 infected controls, i.e $50.1 \%$. Cases and controls were matched for age and gender to eliminate bias.

Further 14 COVID-19 infection confirmed burn cases were compared with 14 controls identified from non-COVID time period, to assess the effect of COVID-19 infection on mortality in burns. Cases and controls were matched for age, gender, and TBSA of burns to eliminate bias. Two cases of COVID-19 infected burns were of pediatric age-group who refused to continue treatment and, hence, could not be followed up. No increased risk of mortality in COVID-19 infected burn cases was seen in comparison to controls (odds ratio $=1$, $p=1.00$ ). Both cases and controls had same mortality rate of $14.3 \%$, i.e 2 out of 14 .

\section{Discussion}

Burn injuries with TBSA of burns $>15 \%$ or when sustained in high-risk age groups like elderly and children require emergency hospital admission without delay. It is after threshold size of $15 \%$ burns that systemic spillover of cytokines start, stimulating the brain to release catecholamines and glucocorticoids causing SIRS. During SIRS in burns, bone marrow is in a state of emergency granulopoiesis with reciprocal reduction in lymphopoiesis and hematopoiesis. Upregulation of myeloid derived suppressor cells leads to immunosuppression. ${ }^{2}$ It is important to understand the pathophysiology of COVID-19 infection, that is, severe acute respiratory syndrome coronavirus 2 (SARS-CoV-2) virus, for assessing its impact in burn patients who are highly vulnerable to such infections.

SARS-COV2 is a single-stranded RNA virus. It spreads by attaching to the nasopharyngeal mucosa, followed by viral replication and transport to lungs causing lower respiratory tract infection. Viral RNA dictates host cell machinery for genome replication and polypeptide synthesis. Spike glycoprotein S promotes virus entry through the angiotensin-converting 
enzyme 2 receptors expressed in lungs, intestines, kidney, brain, and blood vessels. These receptors are negative regulator of the renin angiotensin system involved in inflammation. ${ }^{3,4}$ Therefore, COVID-19 infection in burns can, not only add to the latter's morbidity and mortality but also necessitate the requirement of longer intensive care for such cases. Further, health-care workers involved in burn care are also at a heightened risk in comparison to the general COVID care staff, due to increased contact time with raw burn wounds while dressing and during respiratory care of patients with inhalational burns. ${ }^{5}$

To ensure safety of both patients and doctors from contracting COVID-19 infection, major departmental restructuring of working setup as well as protocols is being done. Setting up of isolation wards, negative pressure operation theaters, screening areas, and ensuring continuous supply of personal protection equipment, N-95 masks, face shields, sanitizers, disinfectants, fumigants, etc. is highly resource consuming. ${ }^{6-8}$ Limited elective operations will not work well for both waiting patients and doctors in the long run. Hence, we need to assess the true impact of COVID-19 infection on patient trends and outcomes, presenting to a burn and plastic unit, especially burns. At the same time, we need to identify risk factors for COVID-19 infection in burn patients. This will help us understand the true epidemiology of COVID-19 infection in burns and help us formulate future planning and management for COVID-19 infection in burns in an economical and resource friendly manner.

Our study recorded a decline in new admissions to the burn and plastic unit by $37.7 \%$ in 2020 in comparison to 2019. Similarly, university hospital at Birmingham recorded 50\% decrease in patients admitted to burn ward. ${ }^{9}$ They observed no change in epidemiology of burns during pandemic with flame burns being the most common. They had no mortality, with $70 \%$ patients having TBSA of burns below $5 \%$. They had no COVID-19-positive patient intake. ${ }^{9}$ Another study from a regional burn center of United States noticed 13\% fall in adult admissions with $9 \%$ increase in pediatric admissions. They recorded no change in patient trends during the pandemic and had no COVID-19-positive burn patient. ${ }^{10}$ Similar results of no change in burn epidemiology during the pandemic were observed in burn centers at Toronto and Northern Israel. They also observed a slight reduction in new admissions with a male preponderance. Their majority cases were of flame burns. However, these centers also did not have any COVID-19-positive burns. ${ }^{11,12}$

The drop in new admissions during the pandemic period can be attributed to lockdown restrictions and fear among patients from coming to hospitals. This has also led to patients landing late in the hospital after worsening of their condition, highlighting the need for stricter transfer guidelines for burn patients. ${ }^{1}$ The number of pediatric cases did not rise in our unit during the pandemic. The mean TBSA of burns is around $42 \%$ in our unit during both study time periods. Thus, our unit has a higher mortality rate in comparison to other centers. Thermal burns continued to be the most common cause of admission even in our study. However, we had more trauma admissions during the pandemic owing to the need for admitting them for COVID-19 testing before any intervention. Most trauma cases were earlier managed on day care basis (-Fig. 1). Males contributed to majority of affected patients in our study. Thermal burns due to LPG leakage at home and fire at workplace, scalds due to contact with hot objects at work and hot fluids at home, electric burns due to contact with high tension wire at workplace, or overhead wiring at homes and vitriolage or industrial exposure for chemical burns continued to be the mechanism of injury even during the pandemic. However, during this pandemic we identified a new mechanism of burns sustained due to use of sanitizer on hand and body followed by cooking. There has been a similar case report of burn in a man due to lighting of cigarette after using hand sanitizer gel. ${ }^{13}$ So, we need to spread awareness about the safe use of sanitizers.

Our study had a total intake of 18 COVID-19-positive patients (burn patients $=16$, trauma patients $=2$ ) of the total 350 new admissions of burns and trauma, over a period of 5 months. Similar study conducted in Padua University Hospital, Italy, identified only two COVID-19-positive patients out of 35 new burn admissions over the 4-month period. ${ }^{14}$ However, epidemiological reports from few other major burn centers during this pandemic, reported no COVID-19-positive burn patient as discussed earlier. ${ }^{9-11}$ Thus, this study with 16 COVID-19-positive burn cases, which is a good number from a single center, provides good insight for the assessment of TBSA of burns as a risk factor for COVID-19 infection in burns. The impact of COVID-19 infection on burns mortality is also evaluated.

On case-control analysis of 16 cases with 16 matched controls for age and gender from COVID times, no increased risk of COVID-19 infection in burn patients with increasing TBSA of burns was seen $(p=0.126)$. Around $50 \%$ cases and controls had TBSA of burns greater than $25 \%$. It was expected that patients with TBSA of burns greater than $25 \%$ will be at higher risk due to immunocompromised state. ${ }^{2}$ This was a matter of concern because around $60 \%$ of our burn admissions during COVID and non-COVID time period had TBSA of burns greater than 25\%. However, no risk of increased COVID-19 infection with higher TBSA of burns was seen in our study.

Two COVID-19-positive burns were of pediatric age group and refused to continue treatment. There were two deaths among the remaining 16 cases. The main reason was acute respiratory distress syndrome in one case and head injury with dyselectrolytemia and pleural effusion in the other. It is difficult to attribute COVID-19 infection solely for the death of these two cases. The infection is expected to have added to the insult of burns by accelerating the inflammatory processes. However, on case-control analysis of these 14 COVID-19-positive cases with identified matched controls for age, gender, and TBSA of burns from non-COVID time period, no significant difference in outcome as mortality was seen $(p=1.00$, odds ratio=1.00). Both groups had same mortality rate of $14.3 \%$ (2 out of 14 ). 
To the best of our knowledge, there is a paucity of studies published till now reporting analysis of risk factors and outcome of COVID-19 infection in burn patients. The limitations of our study include reliance on RT-PCR testing solely for diagnosis of COVID-19 infection. Thus, some of the asymptomatic cases of COVID-19 infection might have been missed in our study. It has been seen that sensitivity of RT-PCR is less than $70 \%$ and it also depends on the sampling technique. ${ }^{15}$ Instead, baseline chest computed tomography (CT) with RT-PCR COVID testing serves as a better and more reliable indicator of the pathology. ${ }^{5}$ Due to our center limitations, CT could not be done for all patients. Also, this is a single-center study with only 16 cases and thus, the results cannot be generalized to the whole population

\section{Conclusion}

This study finds no difference in the trends of new admissions to burn and plastic unit during the pandemic. Among burn patients, no increased risk of COVID-19 infection is seen with larger TBSA of burns. No increase in mortality is seen in burn patients infected with COVID-19. Larger multicenter, case-control observational studies are needed with a larger sample size to establish the definitive risk factors and effect of COVID-19 infection in burns.

\section{Previous presentation in conferences \\ None.}

\section{Funding}

This study did not receive any specific grant from funding agencies in the public, commercial, or not-for-profit sectors.

\section{Conflict of Interest}

None to declare.

\section{Acknowledgments}

None.

\section{References}

1 Kamolz LP, Schiefer JL, Horter J, Plock JA. COVID-19 and burns: lessons learned? Burns 2020;46(6):1467-1468. doi:10.1016/j. burns.2020.05.015.
2 Greenhalgh DG. Sepsis in the burn patient: a different problem than sepsis in the general population. Burns Trauma 2017;5. doi 2310.1186/s41038-017-0089-5.

3 Hesamirostami M, Nazarian R, Asghari H, et al. A case series of concomitant burn and COVID-19. Burns Open 2021;5(1):3438. doi:10.1016/j.burnso.2020.11.003

4 Kumar M, Al Khodor S. Pathophysiology and treatment strategies for COVID-19. J Transl Med 2020;18(1):353. doi:10.1186/ s12967-020-02520-8.

5 Huang Z, Zhuang D, Xiong B, Deng DX, Li H, Lai W. Occupational exposure to SARS-CoV-2 in burns treatment during the COVID-19 epidemic: Specific diagnosis and treatment protocol. Biomed Pharmacother 2020;127:110176. doi:10.1016/j. biopha.2020.110176.

6 Saha S, Kumar A, Dash S, Singhal M. Managing burns during COVID-19 outbreak. J Burn Care Res 2020;41 (5):1033-1036. doi:10.1093/jbcr/iraa086

7 Soltany A, Hasan AR, Mohanna F. Burn management during the COVID-19 pandemic: recommendations and considerations. Avicenna J Med 2020;10(4):163-173. doi:10.4103/ajm. ajm_153_20

8 Li N, Liu T, Chen H, et al. Management strategies for the burn ward during COVID-19 pandemic. Burns 2020;46(4):756-761. doi:10.1016/j.burns.2020.03.013.

9 Farroha A. Effects of COVID-19 pandemic on burns epidemiology. Burns 2020;46(6):1466-1485. doi:10.1016/j. burns.2020.05.022.

10 Williams FN, Chrisco L, Nizamani R, King BT. COVID-19 related admissions to a regional burn center: the impact of shelter-inplace mandate. Burns Open 2020;4(4):158-159. doi:10.1016/j. burnso.2020.07.004.

11 Rogers AD, Cartotto R. The Impact of COVID-19 on Burn Care at a Major Regional Burn Center. J Burn Care Res. 2021;42(1):110111. doi: $10.1093 / \mathrm{jbcr} / \mathrm{iraa} 181$.

12 Kruchevsky D, Arraf M, Levanon S, Capucha T, Ramon Y, Ullmann Y. Trends in Burn Injuries in Northern Israel During the COVID-19 Lockdown. J Burn Care Res. 2021;42(2):135140. doi: $10.1093 / \mathrm{jbcr} / \mathrm{iraa} 154$.

13 Amjadi M, Greenwood JE. Burn injury caused by hand sanitizing gel. J Burn Care Res 2010;31(6):967. doi: 10.1097/ BCR.0b013e3181f93b5b.

14 Azzena B, Perozzo FAG, Lazzari A, De, Valotto G, Pontini A, Burn unit admission and management protocol during COVID19 pandemic. Burns 2020; S0305-4179(20)30525-8. doi: 10.1016/j.burns.2020.09.004

15 Toh VV, Antrum JHG, Sloan B, Austin O, Muthayya P. Management of COVID-19 in burns patients: the experience of a UK burn centre. Burns 2020;46:1707-1728. doi:10.1016/j. burns.2020.06.026. 